\title{
Fator de resposta do alecrim-pimenta a diferentes lâminas de irrigação ${ }^{1}$
}

\author{
Ivan Caldeira Almeida Alvarenga ${ }^{2}$, Otávio Diniz Lopes ${ }^{3}$, \\ Fernanda Ventorim Pacheco ${ }^{4}$, Flávio Gonçalves Oliveira ${ }^{5}$, Ernane Ronie Martins ${ }^{5}$
}

\begin{abstract}
Response of Lippia sidoides

to different irrigation depths

Evaluating the response of medicinal plants to irrigation is not very common, especially when they are native species with economic potential, such as the Lippia sidoides. Thus, this study aimed at evaluating the Lippia sidoides response, by using the Ky coefficient, and growth, under different irrigation depths. The coefficients were determined from the dry matter and essential oil yields. The experiment used a randomized blocks design, with treatments consisting of seven irrigation depths $(0.5,0.75,1.0$, $1.2,1.4,1.65$ and 1.9 of the Eto), with three replications. The ETo was evaluated by using the Penman-Monteith method and the ETr according to the soil water balance. Growth was determined by measuring the stem diameter and height, at 35, 56, 77, 98 and 119 days after transplanting. At 120 days of cultivation, samples were collected to determine the dry matter and essential oil yields. The plant responded to water deficit with coefficients above 1.0, for both dry matter and essential oil yields. Growth was positively affected by irrigation, reaching higher values for stem diameter and height when the highest irrigation depths were used.
\end{abstract}

KEY-WORDS: Lippia sidoides Cham.; medicinal plants; essential oil; water availability.

\section{INTRODUÇÃO}

O desenvolvimento vegetal é influenciado pela disponibilidade de nutrientes, luz, temperatura e água, em proporções adequadas. Este conjunto de fatores, aliado à genética da planta cultivada, determinará a produtividade de uma cultura, promovendo a queda ou o aumento da produção (Andrade \& Casali 1999).

A água é fator limitante à produção agrícola, sendo necessária ao pleno crescimento e desenvolvimento da cultura. Muitas vezes, a água proveniente

\section{RESUMO}

A resposta de plantas medicinais, em relação à irrigação, ainda é pouco estudada, principalmente quando se trata de espécies nativas com potencial econômico, como é o caso do alecrim-pimenta. Assim, este trabalho objetivou determinar a sensibilidade do alecrim-pimenta, por meio do coeficiente $\mathrm{Ky}$, e o seu crescimento, sob diferentes lâminas de irrigação. Os coeficientes foram determinados a partir das produtividades de matéria seca e óleo essencial. O experimento foi conduzido em blocos casualizados, sendo os tratamentos sete lâminas de irrigação $(0,5 ; 0,75 ; 1,0 ; 1,2 ; 1,4 ; 1,65$; e 1,9 da ETo), com três repetições. A ETo foi avaliada pelo método de PenmanMonteith e a ETr de acordo com o balanço hídrico no solo. O crescimento foi acompanhado com medições do diâmetro do coleto e altura, aos 35, 56, 77, 98 e 119 dias após o transplantio. Aos 120 dias de cultivo, realizou-se a coleta das amostras, para determinação da produtividade de matéria seca e óleo essencial. A planta apresentou coeficientes de resposta ao déficit hídrico acima de 1,0, tanto para a produção de matéria seca quanto de óleo essencial. O crescimento da cultura foi influenciado, positivamente, pela irrigação, apresentando maiores valores em diâmetro do coleto e altura para as maiores lâminas aplicadas.

PALAVRAS-CHAVE: Lippia sidoides Cham.; plantas medicinais; óleo essencial; disponibilidade hídrica.

de precipitação é suficiente para a obtenção de uma produtividade adequada, todavia, em locais onde a precipitação é deficitária, ou irregular, a necessidade hídrica é complementada por meio de irrigação. Como a necessidade hídrica varia entre as espécies, e ao longo do seu ciclo, conhecer as respostas das espécies é de grande importância para a elaboração de planos de manejo adequados, considerando-se o uso racional dos recursos disponíveis, de maneira a se obter rendimentos econômicos mais altos (Monteiro et al. 2006, Lima et al. 2012).

1. Trabalho recebido em jun./2012 e aceito para publicação em dez./2012 (nº registro: PAT 19037).

2. Universidade Federal de Lavras (UFLa), Departamento de Fitotecnia, Lavras, MG, Brasil. E-mail: pytchouai@yahoo.com.br.

3. Universidade Estadual de Montes Claros (Unimontes), Janaúba, MG, Brasil.E-mail: otaviodl@gmail.com.

4. Universidade Federal de Lavras (UFLa), Departamento de Biologia, Lavras, MG, Brasil. E-mail: fventorimpacheco@yahoo.com.br. 5. Universidade Federal de Minas Gerais (UFMG), Instituto de Ciências Agrárias, Montes Claros, MG, Brasil.

E-mails: flaviogoliveira@ibest.com.br, ernane.ufmg@gmail.com. 
Como a deficiência hídrica está diretamente ligada à evapotranspiração da cultura, Doorenbos \& Kassam (1979), com base nesta relação, elaboraram um fator de resposta da cultura ao estresse hídrico (Ky), pelo qual se pode determinar em que fase fenológica a planta é mais sensível. Este fator decorre da redução na produtividade relativa, de acordo com a diminuição da evapotranspiração da cultura, causada pelo déficit hídrico. De acordo com Raes et al. (2006), o fator de resposta Ky apresenta-se como importante ferramenta para o cultivo irrigado, e foi por eles utilizado para comparar métodos de manejo de irrigação de milho e trigo.

O alecrim-pimenta (Lippia sidoides Cham.) é uma espécie aromática e medicinal, originária do Nordeste brasileiro e de regiões semiáridas do norte de Minas Gerais. O potencial econômico da planta deve-se ao seu óleo essencial, composto, majoritariamente, por timol e carvacrol, apresentando propriedades antissépticas e antimicrobianas (contra fungos e bactérias), atividade antihelmíntica e efeito gastroprotetor e larvicida (Alvarenga et al. 2009).

Tendo-se em vista a importância medicinal da espécie, e de se conhecer o comportamento da cultura sob cultivo irrigado, este estudo objetivou determinar o fator de resposta (Ky) do alecrim-pimenta, em relação à produtividade de matéria seca e óleo essencial, bem como o crescimento da cultura, sob sete lâminas de irrigação.

\section{MATERIAL E MÉTODOS}

O experimento foi conduzido em lisímetros de drenagem, instalados a campo e a céu aberto, em área experimental do Instituto de Ciências Agrárias da Universidade Federal de Minas Gerais (UFMG), em Montes Claros, MG, de 15 de maio a 15 de setembro de 2009. O clima, no local, é do tipo Aw, segundo a classificação de Köppen (Leite et al. 2004), caracterizando-se como tropical de savana, com inverno seco e verão chuvoso, semiárido.

Os lisímetros consistiram em caixas de polietileno, com capacidade de $1,0 \mathrm{~m}^{3}$, enterradas a até
$10,0 \mathrm{~cm}$ da borda e preenchidas com 950,0 kg de solo seco. Cada lisímetro foi conectado a um coletor, por meio de tubulação de PVC (1"/2), podendo-se, então, aferir a lâmina percolada. O solo utilizado para o cultivo foi classificado como Latossolo Vermelho-Amarelo (Santos et al. 2006) de textura argilosa e sua caracterização físico-química (Tabela 1) foi realizada no laboratório de solos da mesma instituição onde o experimento foi conduzido.

O manejo de irrigação e a determinação da evapotranspiração da cultura $(\mathrm{ETc})$ foram realizados a partir do balanço hídrico no solo, conforme a equação de Reichardt (1985): P + I - D - ETc $= \pm \Delta$ h, onde $\mathrm{P}=$ precipitação natural; $\mathrm{I}=$ irrigação; $\mathrm{D}=$ drenagem profunda; $\mathrm{ETc}=$ evapotranspiração da cultura; e $\Delta \mathrm{h}=$ variação da armazenagem da água no solo, dentro dos lisímetros.

A ETo foi obtida a partir da equação de Penman-Monteith (Allen et al. 1998), sendo utilizados os dados obtidos em estação meteorológica automática (modelo Davis Pro 2), à distância de 10,0 m, em relação ao experimento ( $16^{\circ} 44^{\prime} 02^{\prime \prime} \mathrm{S}, 43^{\circ} 51^{\prime} 23^{\prime}$ 'W).

Os tratamentos consistiram de sete lâminas de irrigação $(0,5 ; 0,75 ; 1,0 ; 1,2 ; 1,4 ; 1,65$ e 1,9 da ETo calculada, denominadas T1, T2, T3, T4, T5, T6 e T7, respectivamente), com três repetições, distribuídas em blocos inteiramente casualizados, perfazendo 21 unidades experimentais. O solo de cada parcela experimental foi saturado e as caixas foram tampadas, para impedir a evaporação da água, a fim de se obter a capacidade de campo, após a drenagem total, a qual ocorreu três dias após a saturação, permitindo o início do experimento, para a realização do balanço hídrico.

Com o solo na capacidade de campo, as mudas de alecrim-pimenta foram transplantadas em covas com espaçamento de $0,4 \mathrm{~m}$ entre as fileiras e $0,4 \mathrm{~m}$ entre as plantas, na linha, formando uma área por planta de $0,16 \mathrm{~m}^{2}$, com nove unidades por lisímetro (três fileiras, com três plantas cada), totalizando 189 plantas. Foi realizada uma adubação orgânica, na proporção de $12,0 \mathrm{~kg} \mathrm{~m}^{-2}$, com esterco bovino curtido, 15 dias antes do plantio (Assis et al. 2009). Aos 120 dias de cultivo, foram coletadas amostras,

Tabela 1. Características físico-químicas do solo utilizado no experimento.

\begin{tabular}{|c|c|c|c|c|c|c|c|c|c|}
\hline $\mathrm{pH}$ & P. rem. & $\mathrm{Ca}$ & $\mathrm{Mg}$ & $\mathrm{Al}$ & $\mathrm{V}$ & Areia & Silte & Argila & M.O. \\
\hline $\mathrm{H}_{2} \mathrm{O}$ & $\mathrm{mg} \mathrm{kg}^{-1}$ & 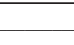 & $\mathrm{mol} \mathrm{c}^{\circ}$ & - & $\%$ & 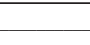 & - & 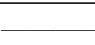 & 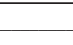 \\
\hline 5,0 & 78 & 1,4 & 0,30 & 2,36 & 22 & 44,0 & 20,0 & 36,0 & 1,35 \\
\hline
\end{tabular}


para determinar a produtividade de matéria seca e óleo essencial.

Na determinação da matéria seca, amostras de $100,0 \mathrm{~g}$ de material fresco foram colocadas em estufa de circulação forçada, por 72 horas, à temperatura constante de $60^{\circ} \mathrm{C}$, e, posteriormente, pesadas em balança de precisão.

$\mathrm{Na}$ determinação da produção de óleo essencial, as amostras de material fresco $(100,0 \mathrm{~g})$ foram hidrodestiladas, em extrator do tipo Clevenger, durante 4 horas, sendo o óleo obtido pesado em balança analítica. Com base na matéria fresca e na produção de matéria seca, foi estipulada a produtividade do óleo essencial, para cada tratamento.

Para a determinação do Ky, a ETc obtida no balanço hídrico foi considerada como evapotranspiração real da cultura (ETr). Assim, a partir dos dados de produção de óleo e massa seca da parte aérea e dos cálculos de ETr e evapotranspiração máxima da cultura (ETm), foi possível determinar a sensibilidade da cultura do alecrim-pimenta, submetida a diferentes lâminas de irrigação, pelo fator Ky, a partir da equação elaborada por Doorenbos \& Kassam (1994): $\mathrm{Yr} / \mathrm{Ym}=\mathrm{Ky}(1-\mathrm{ETr} / \mathrm{ETm})$, onde $\mathrm{Yr}=$ produtividade real da cultura; $\mathrm{Ym}=$ produtividade máxima da cultura; Ky = fator de resposta produtiva ao déficit hídrico; ETr = evapotranspiração real da cultura; e $\mathrm{ETm}=$ evapotranspiração máxima da cultura.

O crescimento das plantas foi acompanhado por meio de determinações da altura e diâmetro do colo, sendo feitas medições aos 35, 56, 77, 98 e 119 dias após o transplantio. Na determinação da altura, mediu-se o comprimento da base do caule até a extremidade do ramo mais alto, por meio de trena graduada com precisão de $1,0 \mathrm{~mm}$, e o diâmetro do caule, tomando-se a medida a $2,0 \mathrm{~cm}$ do solo, com paquímetro digital com precisão de $0,01 \mathrm{~mm}$. Os dados de produção de massa seca da parte aérea, produtividade de óleo e crescimento foram submetidos à análise de regressão, pelo programa SAEG (UFV 1997).

\section{RESULTADOS E DISCUSSÃO}

Observaram-se temperaturas máximas entre $30^{\circ} \mathrm{C}$ e $35^{\circ} \mathrm{C}$ e mínimas entre $10^{\circ} \mathrm{C}$ e $15^{\circ} \mathrm{C}$, nos últimos 30 dias de cultivo (Figura 1a). A evapotranspiração de referência apresentou comportamento crescente, com valores máximos de até $6,0 \mathrm{~mm} \mathrm{dia}^{-1}$, devido ao progresso da estação de estiagem, sendo que a evapotranspiração média foi de $4,0 \mathrm{~mm} \mathrm{dia}^{-1}$ (Figura 1b). Comparativamente, observou-se declínio na umidade relativa do ar, apresentando mínimas em torno de $40 \%$, aos 112 dias de cultivo, sendo que o valor médio, durante o período, foi de $60 \%$ (Figura 1c). Estas variáveis meteorológicas observadas concordam com o tipo de clima descrito para a região $(\mathrm{Aw})$. A espécie analisada apresenta comportamento caducifólio (Carvalho Júnior et al. 2011), contudo, no período de condução do trabalho (maio a setembro), não foi observado este com-
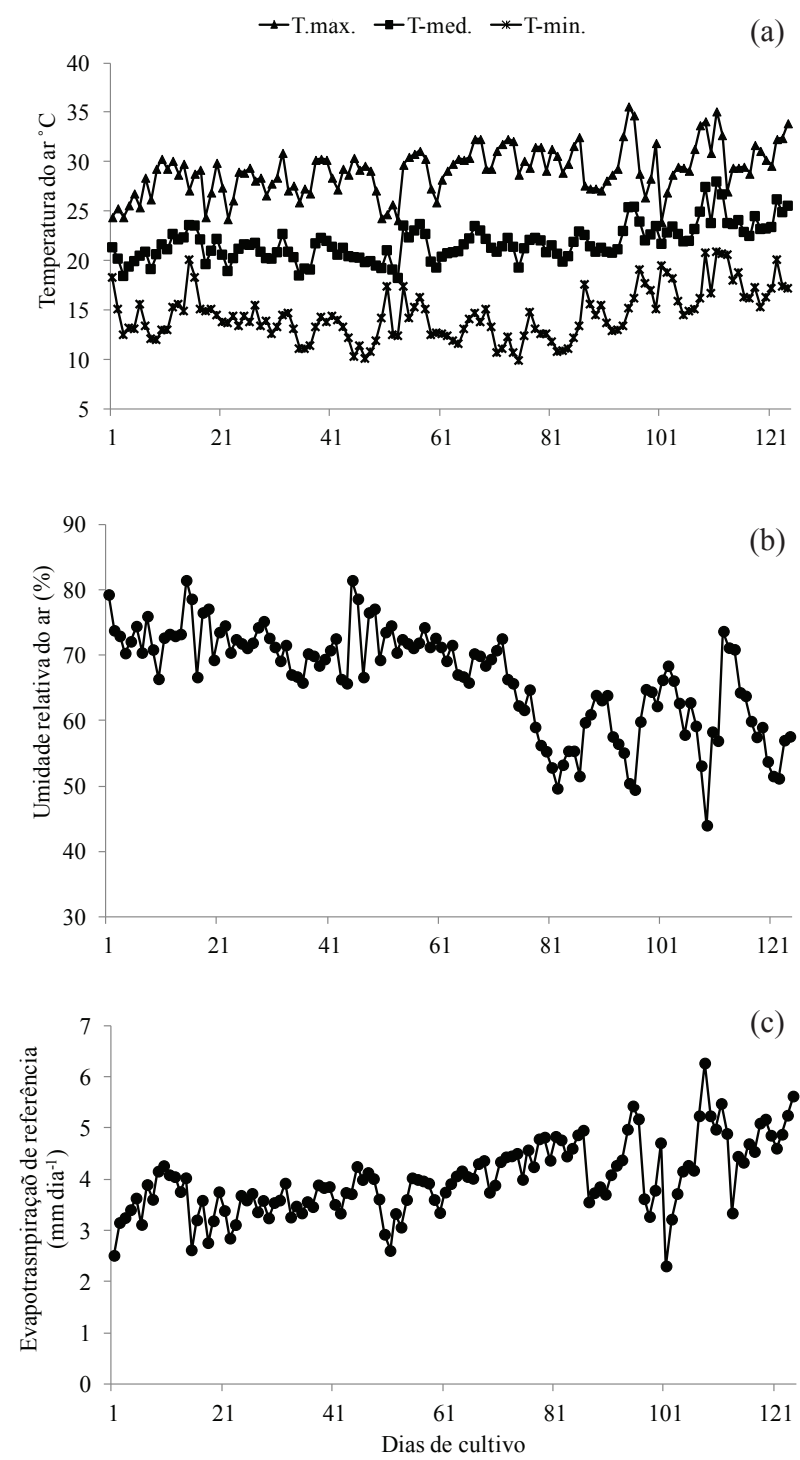

Figura 1. Oscilação das temperaturas máximas (T.max.), médias (T.med.) e mínimas (T.min.) (a), da umidade relativa do ar (b) e da evapotranspiração de referência (PenmanMonteith) (c), durante o período de cultivo de alecrimpimenta (Montes Claros, MG, 2009). 
portamento, mesmo nos tratamentos com menores lâminas de água, demostrando que a permanência das folhas pode estar relacionada com a disponibilidade hídrica.

Pôde-se observar a evolução do crescimento em altura e diâmetro das plantas, ao longo do ciclo de cultivo, bem como as equações ajustadas, significativas pelo teste $\mathrm{F}(\mathrm{p}<0,05 \%)$, com o maior crescimento ocorrendo na maior disponibilidade hídrica, aos 119 dias de cultivo (Figura 2). Estes resultados estão de acordo com o estudo conduzido por Figueiredo et al. (2009), para a mesma espécie e local de estudo, no qual observou-se média de altura de $180,0 \mathrm{~cm}$ e diâmetro do caule próximo a $11,0 \mathrm{~mm}$, porém, com o ciclo de cultivo de 300 dias, em cultivo irrigado não manejado. Assim, pôde-se inferir que a maior oferta hídrica acelerou o crescimento da planta.
Para a produtividade de óleo essencial, com base na matéria seca (Figura 3a), observou-se que, com o aumento da disponibilidade hídrica, ocorreu, também, maior produção de óleo essencial, indicando que a sua produção está ligada à disponibilidade hídrica. Os valores variaram entre $6,78 \mathrm{~kg} \mathrm{ha}^{-1}$ e $116,98 \mathrm{~kg} \mathrm{ha}^{-1}$, para a menor e maior lâminas consumidas, respectivamente. Comparativamente, para manjericão (Ocimum basilicum L.), Pravuschi et al. (2010) observaram produtividades entre $26,68 \mathrm{~kg} \mathrm{ha}^{-1}$ e $58,25 \mathrm{~kg} \mathrm{ha}^{-1}$, com a maior produtividade sendo obtida com a reposição de $100 \%$ da evaporação do tanque Classe A, corroborando as conclusões de Marques et al. (2009), que, para orégano (Origanum vulgaris), também observaram maiores rendimentos de óleo e matéria seca, para o mesmo tratamento.

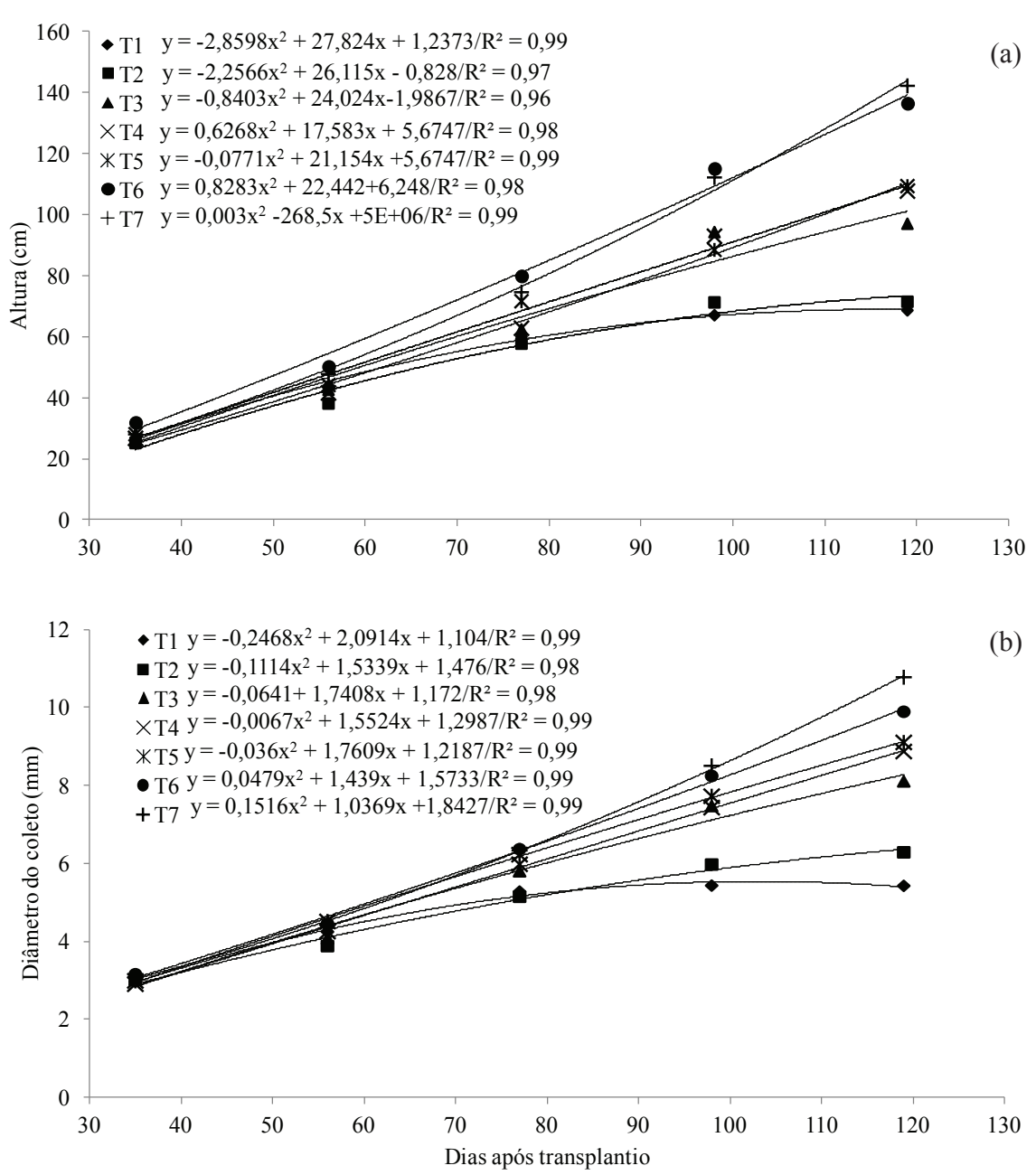

Figura 2. Crescimento em altura (a) e em diâmetro do coleto (b) de alecrim-pimenta, em função de diferentes evapotranspirações reais da cultura (Montes Claros, MG, 2009). T1: 267,5 mm; T2: 346,5 mm; T3: 427,5 mm; T4: 470,9 mm; T5: 491,3 mm; T6: 543,3 mm; e T7: 578,3 mm. 

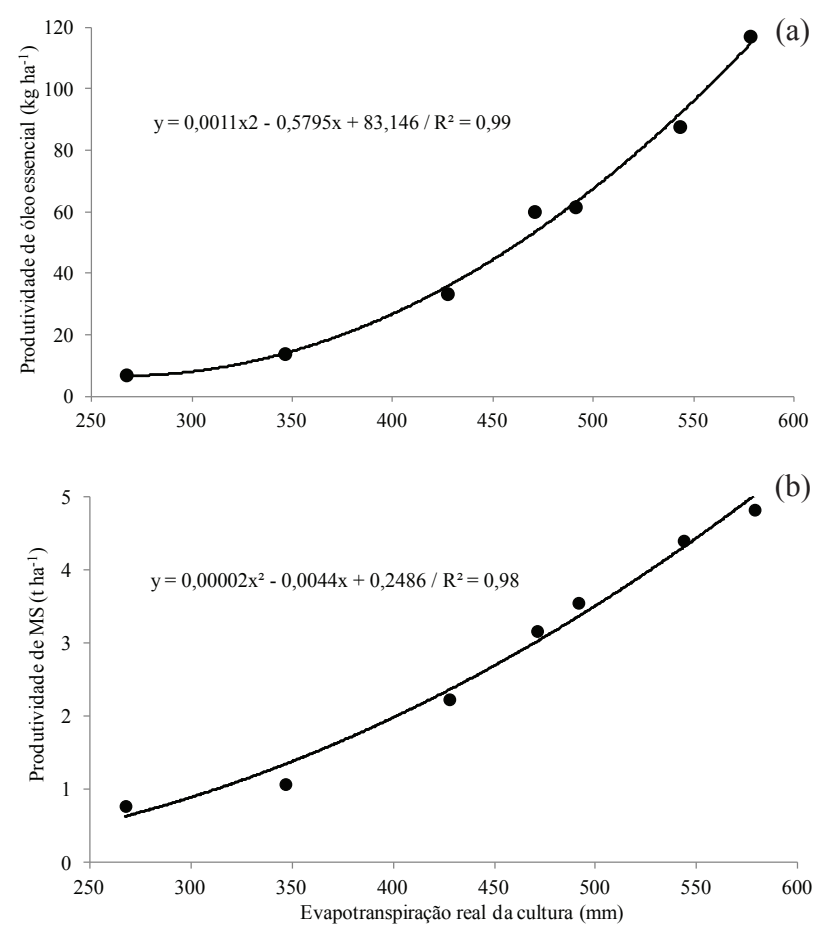

Figura 3. Produtividade de óleo essencial (a) e matéria seca (b) de alecrim-pimenta, em função de diferentes evapotranspirações reais da cultura (Montes Claros, MG, 2009).

Para boldo (Peumus boldus), Vogel et al. (2011) observaram maiores teores de óleo essencial, com o solo mantido a $65 \%$ da capacidade de campo, quando comparado com o tratamento sob estresse, a $25 \%$ da capacidade de campo.

A produtividade de matéria seca foi favorecida pelo fornecimento de água (Figura 3b), com valores variando de $0,76 \mathrm{t} \mathrm{ha}^{-1}$ até o valor máximo de 4,42 tha ${ }^{-1}$, com a evapotranspiração da cultura de $578,32 \mathrm{~mm}$. Estes resultados corroboram os observados por Costa Filho et al. (2006), os quais constataram que o solo, mantido a $100 \%$ da capacidade de campo, promoveu a maior alocação de matéria seca, em
Ocimum gratissimum, acelerando o desenvolvimento da planta. As maiores lâminas aplicadas favoreceram a produção, provavelmente, devido à solubilização dos nutrientes do solo e manutenção do equilíbrio na transpiração e fotossíntese (Khan et al. 2012).

As determinações dos valores de Ky tiveram como base de cálculo a maior produtividade, tanto de matéria seca quanto de óleo, com ETr de $578,32 \mathrm{~mm}$. O valor máximo de Ky, para a matéria seca, foi de 2,07 (Tabela 2), valor encontrado para a evapotranspiração real de 427,49 mm, próximo à capacidade de campo do solo, sendo que, para as lâminas consumidas superiores a esta, os valores de resposta foram menores.

Isso pode estar relacionado à capacidade de a planta se adaptar a oscilações hídricas, de modo a ajustar os processos metabólicos, de tal forma que, a partir de determinado status hídrico do solo, a resposta produtiva não seja afetada (Larcher 2000). Para a produtividade de óleo (Tabela 3), o valor de Ky foi máximo para a ETr de $543,37 \mathrm{~mm}$, podendo-se inferir que a produção do óleo esteja ligada à disponibilidade hídrica (Figura 2a).

Gomes \& Carr (2003), em estudos com batata doce (Ipomea batatas), observaram aumento nos valores de Ky de 0,9 para 1,2, quando aumentada a frequência de colheita, indicando a necessidade hídrica da espécie, neste tipo de manejo. Em cultivo de melancia (Citrullus lunatus), as fases de maior sensibilidade hídrica foram o desenvolvimento inicial e a floração, com valores de Ky em torno de 0,66 , no entanto, avaliando-se todo o ciclo, a cultura apresentou baixa sensibilidade ao déficit hídrico, com índices inferiores a 1,0 (Freitas et al. 1999).

Para Doorenbos \& Kassam (1979), a classificação das culturas, quanto à sensibilidade ao estresse hídrico, pode ser dividida em quatro categorias: baixa $(\mathrm{Ky}<0,85)$, baixa/média $(0,85<\mathrm{Ky}<1,00)$, média/ alta $(1,00<\mathrm{Ky}<1,15)$ e alta $(\mathrm{Ky}>1,15)$. Assim,

Tabela 2. Valores de evapotranspiração real da cultura (ETr), evapotranspiração máxima da cultura (ETm), produtividade real da cultura (Yr), produtividade máxima da cultura (Ym) e coeficiente de sensibilidade (Ky), para a produtividade de matéria seca $\left(\mathrm{t} \mathrm{ha}^{-1}\right)$ de alecrim-pimenta (Montes Claros, MG, 2009).

\begin{tabular}{cccccccccc}
\hline Tratamentos & $\mathrm{ETr}$ & $\mathrm{ETm}$ & $\mathrm{ETr} / \mathrm{ETm}$ & $1-(\mathrm{ETr} / \mathrm{ETm})$ & $\mathrm{Yr}$ & $\mathrm{Ym}$ & $\mathrm{Yr} / \mathrm{Ym}$ & $1-(\mathrm{Yr} / \mathrm{Ym})$ & $\mathrm{Ky}$ \\
\hline 1 & 267,50 & 578,32 & 0,46 & 0,54 & 0,76 & 4,83 & 0,16 & 0,84 & 1,57 \\
2 & 346,50 & 578,32 & 0,60 & 0,40 & 1,09 & 4,83 & 0,23 & 0,77 & 1,93 \\
3 & 427,49 & 578,32 & 0,74 & 0,26 & 2,23 & 4,83 & 0,46 & 0,54 & 2,07 \\
4 & 470,86 & 578,32 & 0,81 & 0,19 & 3,18 & 4,83 & 0,66 & 0,34 & 1,84 \\
5 & 491,25 & 578,32 & 0,85 & 0,15 & 3,51 & 4,83 & 0,73 & 0,27 & 1,81 \\
6 & 543,27 & 578,32 & 0,94 & 0,06 & 4,42 & 4,83 & 0,92 & 0,08 & 1,39 \\
\hline
\end{tabular}


Tabela 3. Valores de evapotranspiração real da cultura (ETr), evapotranspiração máxima da cultura (ETm), produtividade real da cultura (Yr), produtividade máxima da cultura (Ym) e coeficiente de sensibilidade (Ky), para a produtividade de óleo essencial $\left(\mathrm{kg} \mathrm{ha}^{-1}\right)$ de alecrim-pimenta (Montes Claros, MG, 2009).

\begin{tabular}{cccccccccc}
\hline Tratamentos & $\mathrm{ETr}$ & $\mathrm{ETm}$ & $\mathrm{ETr} / \mathrm{ETm}$ & $1-(\mathrm{ETr} / \mathrm{ETm})$ & $\mathrm{Yr}$ & $\mathrm{Ym}$ & $\mathrm{Yr} / \mathrm{Ym}$ & $1-(\mathrm{Yr} / \mathrm{Ym})$ & $\mathrm{Ky}$ \\
\hline 1 & 267,50 & 578,32 & 0,46 & 0,54 & 6,8 & 117,0 & 0,06 & 0,94 & 1,753 \\
2 & 346,50 & 578,32 & 0,60 & 0,40 & 13,7 & 117,0 & 0,12 & 0,88 & 2,202 \\
3 & 427,49 & 578,32 & 0,74 & 0,26 & 33,2 & 117,0 & 0,28 & 0,72 & 2,746 \\
4 & 470,86 & 578,32 & 0,81 & 0,19 & 59,9 & 117,0 & 0,51 & 0,49 & 2,624 \\
5 & 491,25 & 578,32 & 0,85 & 0,15 & 61,5 & 117,0 & 0,53 & 0,47 & 3,152 \\
6 & 543,27 & 578,32 & 0,94 & 0,06 & 87,6 & 117,0 & 0,75 & 0,25 & 4,148 \\
\hline
\end{tabular}

o alecrim-pimenta mostrou-se uma cultura sensível ao déficit hídrico, apresentando valores médios de Ky superiores a 1,15, sendo eles de 1,57 e 2,77, respectivamente, para a produtividade de matéria seca e óleo essencial.

\section{CONCLUSÕES}

1. O alecrim-pimenta apresentou sensibilidade ao déficit hídrico, para a produção de matéria seca e óleo essencial, com valores médios de Ky de 1,57 e 2,77, respectivamente.

2. A evapotranspiração real da cultura de $578,3 \mathrm{~mm}$, durante um ciclo de 120 dias, aumentou a produtividade de matéria seca e a produtividade de óleo essencial.

3. A maior disponibilidade hídrica favoreceu o desenvolvimento em altura e diâmetro do coleto.

\section{AGRADECIMENTOS}

Ao Conselho Nacional de Desenvolvimento Científico e Tecnológico (CNPq) e à Coordenadoria de Aperfeiçoamento de Pessoal de Nível Superior (Capes), pela concessão de bolsas de mestrado, e à Fundação de Amparo à Pesquisa do Estado de Minas Gerais (Fapemig), pelo apoio financeiro.

\section{REFERENNCIAS}

ALLEN, R. G et al. Crop evapotranspiration: guidelines for computing crop water requirements. Roma: FAO, 1998. (Irrigation and drainage paper, 56).

ALVARENGA, I. C. A. et al. Alecrim-pimenta (Lippia sidoides Cham.): uma espécie aromática e medicinal em domesticação. Caderno de Ciências Agrárias, Montes Claros, v. 1, n. 1, p. 9-25, 2009.
ANDRADE, F. M. C.; CASALI, V. W. D. Plantas medicinais e aromáticas: relação com o ambiente, colheita e metabolismo secundário. Viçosa: UFV, 1999.

ASSIS, B. F. et al. Produção de fitomassa e de óleo essencial de alecrim-pimenta em função da adubação orgânica. Revista Brasileira de Agroecologia, Maringá, v. 4, n. 2, p. 4385-4388, 2009.

CARVALHO JÚNIOR, W. G. O. et al. Fenologia do alecrim-pimenta (Lippia sidoides Cham.) em área de Cerrado, no norte de Minas Gerais, Brasil. Revista Brasileira de Plantas Medicinais, Botucatu, v. 13, n. 2, p. 223-229, 2011.

COSTA FILHO, L. O. et al. Influência hídrica e térmica e desenvolvimento de Ocimum gratissimum L. Revista Brasileira de Plantas Medicinais, Botucatu, v. 8, n. 1, p. 8-13, 2006.

DOORENBOS, J.; KASSAM, A. H. Efeito da água no rendimento das culturas. Campina Grande: UFPB, 1994. (Irrigação e drenagem, 33).

DOORENBOS, J.; KASSAM, A. H. Yield response to water. Rome: FAO, 1979. (Technical note, 33).

FIGUEIREDO, L. S. et al. Efeito da época de colheita na produção de fitomassa e rendimento de óleo essencial de alecrim-pimenta (Lippia sidoides Cham.). Revista Brasileira de Plantas Medicinais, Botucatu, v. 11, n. 2, p. 154-158, 2009.

FREITAS, A. A. et al. Determinação da evapotranspiração máxima e real e do fator de sensibilidade ao déficit hídrico da melancia em Canindé, CE. Revista Brasileira de Engenharia Agrícola e Ambiental, Campina Grande, v. 3, n. 3, p. 298-303, 1999.

GOMES, F.; CARR, M. K. V. Effects of water availability and vine harvesting frequency on the productivity of sweet potato in southern Mozambique: III crop yield/water-use response functions. Experimental Agriculture, Cambridge, v. 39, n. 4, p. 523-537, 2003.

KHAN, M. M. et al. Variations in basil antioxidant contents in relation to deficit irrigation. Journal of Medicinal Plants Research, Nsukka, v. 6, n. 11, p. 2200-2223, 2012. 
LARCHER, W. Ecofisiologia vegetal. São Carlos: RIMA, 2000.

LEITE, G. L. D. et al. Intensidade de ataque de tripes, de alternaria e da queima-das-pontas em cultivares de cebola. Horticultura Brasileira, Brasília, DF, v. 22, n. 1, p. 151-153, 2004.

LIMA, M. E. et al. Desempenho do cultivo da berinjela em plantio direto submetida a diferentes lâminas de irrigação. Revista Brasileira de Engenharia Agrícola e Ambiental, Campina Grande, v. 16, n. 6, p. 604-610, 2012.

MARQUES, P. A. A. et al. Crescimento, produção de óleo essencial e trocas gasosas em orégano influenciados por diferentes lâminas de irrigação. Ciência Rural, Santa Maria, v. 39, n. 6 p. 1888-1892, 2009.

MONTEIRO, R. O. C. et al. Função de resposta do meloeiro a diferentes lâminas de irrigação e doses de nitrogênio. Horticultura Brasileira, Brasília, DF, v. 24, n. 4, p. 455-459, 2006.

PRAVUSCHI, P. R. et al. Efeito de diferentes lâminas de irrigação na produção de óleo essencial do manjericão (Ocimum basilicum L.). Acta Scientiarum Agronomy, Maringá, v. 32, n. 4, p. 687-693, 2010.
RAES, D. et al. Simulation of yield decline as a result of water stress with robust soil water balance model. Agriculture Water Management, Amsterdan, v. 81, n. 3, p. 335-357, 2006.

REICHARDT, K. Processo de transferência no sistema solo-água-atmosfera. 4. ed. Campinas: Fundação Cargill, 1985.

SANTOS, H. G. et al. Sistema brasileiro de classificação de solos. 2. ed. Rio de Janeiro: Embrapa Solos, 2006.

UNIVERSIDADE FEDERAL DE VIÇOSA (UFV). $S A E G$ : sistema de análises estatísticas e genéticas. Versão 9.1. Viçosa: UFV, 1997.

VOGEL, H. et al. Boldo (Peumus boldus) cultivated under different light conditions, soil humidity and plantation density. Industrial Crops and Products, Amsterdan, v. 34, n. 2, p. 1310-1312, 2011. 